\title{
Mechanisms of Action of Noninvasive Monopolar Radiofrequency: Technology Review
}

\author{
Terry L. Whipple ${ }^{1}$, Scott P. Steinmann ${ }^{2}$ \\ ${ }^{1}$ Orthopaedic Research of Virginia, Richmond, USA; ${ }^{2}$ Department of Orthopaedics, Mayo Clinic, Rochester, USA. \\ Email: whipple@americanself.com,steinmann.scott@mayo.edu
}

Received December $4^{\text {th }}, 2012$; revised January $5^{\text {th }}, 2013$; accepted February $6^{\text {th }}, 2013$

\begin{abstract}
Tendinosis is now understood as the result of a failed tendon healing process regardless of where it occurs in the body. Current noninvasive therapeutic alternatives are anti-inflammatory in nature and outcomes are unpredictable at best. The benefit of invasive alternatives resides in the induction of the healing response as demonstrated in pre-clinical and clinical studies in cardiology and orthopaedics. A new technology that employs noninvasivemonopolar capacitive-coupled radiofrequency (mcRF), has demonstrated the ability to raise temperatures in tendons and ligaments upwards of $50^{\circ} \mathrm{C}$, the temperature threshold for collagen modulation and recruitment of macrophages, fibroblasts and Heat Shock Protein factors-without damaging the overlying structures-resulting in activation of the Wound Healing Response (WHR).
\end{abstract}

Keywords: Thermal Shrinkage; Thermoplasty; Heat-Shock; AT2; Radiofrequency; Collagen Denaturation; Tendinosis; Wound Healing Response; Tendinopathy; Microtenotomy; Antinociceptive

\section{Background}

\section{Tendinosis}

In 1951 Bernstein described for the first time a clinical entity of the shoulder as tendinosis [1]. In 1979 Nirschl described the histopathological findings of 88 elbows treated operatively for tennis elbow, with the main finding of immature fibroblastic and vascular infiltration [2] followed by a paper entitled "Elbow Tendinosis/Tennis Elbow," stating that the histology of pathologic tennis elbow tissue revealed non-inflammatory tissue [3].

In 1999 Kraushaar and Nirschl, in a current concepts review for JBJS, concluded that "regardless of what it is called, tendinosis is the result of failed tendon healing" and furthermore that "it can be assumed that tendinosis has essentially the same pathogenesis regardless of where it occurs in the body" [4]. Today common problems of tendons and ligaments (e.g., tennis elbow, golfer's elbow, patellar tendinosis, and plantar fasciosis) are understood as non-inflammatory conditions, and even though some confusion remains in the terminology [5], it is clear that in the lack of inflammation, anti-inflammatory therapeutic options are not indicated (i.e. non-steroidal anti-inflammatory drugs, corticosteroid injections, etc.).

\section{Radiofrequency}

Supra-physiological temperatures are widely used for therapeutic purposes. Radiofrequency (RF) energy is the most commonly used source of energy to generate therapeutic levels of heat. Since its introduction to the field of neurology in the $19^{\text {th }}$ century [6], its applications in medicine have broadened to fields like general surgery, cardiology, neurosurgery, orthopaedics, and ophthalmology [7-16]. In orthopaedics, the main target of supraphysiological temperatures is collagen based connective structures.

\subsection{Non-Biologically Mediated Effects of RF}

\subsubsection{Supra-Physiological Temperatures Effect on Collagen Structure}

An irreversible process results from heating of collagen transforming the original triple helixstructure into a random and contracted structure [17]. This process is time dependent and is better understood under the Arrhenius equation. It is believed that the irreversible process results from the breaking of long sequences of the hydrogen bonds that stabilize the triple helix [18]. The ability of the collagen to reach a contracted phase under these circumstances has been used to shrink and tighten joint stabilizing structures and has become predictably effective. These technological developments offer patients improved joint stability by thermally induced contraction of capsules and ligaments [19-23]. 


\subsubsection{Supra-Physiological Temperatures Effect on Nociceptors}

Patients treated with RF Transmyocardial Revascularization (TMR) had a significant reduction of pain [24]. In a similar finding, $70 \%$ of patients treated with invasive bipolar radiofrequency (bRF) obtained pain relief as early as in the recovery room or on the first or second postoperative day [25]. It is believed that the actual symptoms of tendinosis may be due to biochemical agents irritating nociceptors [26]. This led Takahashi [27] et al. to study the antinociceptive effects of RF concludeing that invasive bRF induced acute degeneration and/or ablation of sensory nerve fibers, explaining the early pain relief associated with these treatments. It has been estab-lished that the antinociceptive effect of RF has clinical utility also in the treatment of plantar fasciosis $[28,29]$.

\subsection{Biologically Mediated Effects of RF}

Desirable and very important biologically mediated effects result from the use of supra-physiological temperatures. Examples of these biologically mediated effects are the induction of molecular and cellular responses, including increased expression of Heat Shock Proteins (HSP), cell death, protein denaturation, and tissue coagulation [30]. HSP have been described to protect cells in vivo and in vitro against diverse insults [31-34].

\subsubsection{Supra-Physiological Temperatures Effect on Myocardium}

RF Trans-Myocardial Revascularization (TMR), a procedure in which radiofrequency is used to create small holes ("channels") in the left ventricle, has shown to enhance angiogenesis and cause myocardial denervation $[35,36]$. TMR improves ischemic symptoms by promoting new vascular networks in treated tissue which become more extensive compared to baseline [24]. Immunochemistry studies showed increased localized presence of basic Fibroblastic Growth Factor (FGF) and Vascular Endothelial Growth Factor (VEGF) at TMR treated sites. These growth factors are known to contribute to endothelial cell migration and angiogenesis, important for an effective WHR [24], significantly reducing pain and improving myocardial function.

\subsubsection{Supra-Physiological Temperatures Effect on Muscle}

Supra-physiological temperatures effect on muscle is mediated by the impact on Myogenic Precursor Cells (MPCs) and is emerging as an effective treatment for some injuries [37]. Rapid, precise application of heat at the injured site improves and accelerates the healing process by stimulating MPCs and protein synthesis and increasing the HSPs [37]. Yang demonstrated that MPCs treated with supra-physiological temperatures induce an overexpression of HSPs [38]. Among the HSP family, HSP70 is the most abundant protein following supra-physiological temperatures [34]. The cytoprotective function of HSP70 has been extensively documented in inflammation, infection and ischemia [31-34].

\subsubsection{Supra-Physiological Temperatures Effect on Tendons and Ligaments}

The understanding of tendinosis as a failed WHR, the positive outcomes of TMR in stimulating the WHR, and the understanding of the capabilities of RF to induce the WHR led Tasto et al. to conduct preclinical studies that demonstrated the feasibility of initiating the WHR in injured tendon tissue utilizing invasive bRF to create similar micro-wounds to those of TMR (microtenotomies) [25]. Later, preclinical and clinical studies by several authors have demonstrated the benefit of inducing the WHR for the treatment of tendinosis [25,39-43].

\section{Noninvasive Monopolar Capacitive-Coupled Radiofrequency (mcRF)}

Noninvasive monopolar capacitive-coupled (mcRF) is made possible by the creation of a reverse thermal gradient and capacitive coupling the energy into a volume of tissue (Figure 1). Noninvasive mcRF was first introduced to plastic surgery as an alternative to reduce the appearance of wrinkles and rhytids [44]. Noninvasive mcRF for this indication has proven to be safe, and adverse events when present are aesthetic in nature (e.g., persistent erythema, blisters, and superficial burns) [45].

\subsection{Noninvasive mcRF in Orthopaedics}

In a similar way, when used in orthopaedics, noninvasive mcRF cools down a volume of tissue (larger than when used for aesthetic indications) while generating an energy

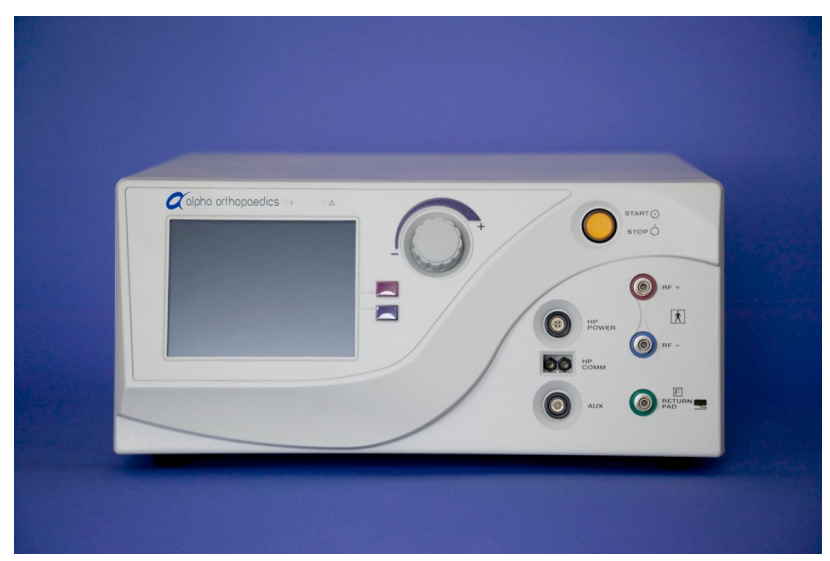

Figure 1. AT2 System mcRF Generator (Alpha Orthopaedics, Inc., Hayward, CA). 
field with a greater depth of penetration than when used for aesthetic applications and only possible via a capacitive coupled electrode (Figure 2).

Noninvasive mcRF for the treatment of conditions of ligaments and tendons benefits from the three main mechanisms of action of RF: the ability of the collagen to reach a contracted phase, the antinociceptive effect, and the ability to stimulate the WHR. The use of noninvasive mcRF may benefit orthopaedic conditions of the muscles via the antinociceptive effect, the ability to stimulate the WHR, and the ability to stimulate MPCs.

\subsection{Preclinical and Clinical Background}

Previous studies in cadaveric specimens have demonstrated that noninvasive mcRF is capable of denaturing the collagen molecules within ligaments and tendons without damaging the skin or subcutaneous layers [46]. These events are known to be followed by an active WHR as demonstrated by the study of England et al. which found that in vivo, the resulting wound healing response from a thermal injury from noninvasive mcRF is largely similar in nature and timing to those of incisional or burn wounds [7]. Anecdotal experience in 2005 with the use of noninvasive mcRF for the treatments of conditions of the tendons and ligaments strongly suggested attainable clinical benefits. Unpublished clinical data [47-49] suggest that clinical outcomes are comparable to those published for the bipolar invasive radiofrequency modality [43].

\section{The Treatment of Tendinosis}

The first goal of the treatment of tendinosis with noninvasive mcRF is to reduce the pain. Pain control can be achieved by blocking nociceptors, since it seems that the actual symptoms of tendinosis are due to irritation of nociceptors by biochemical agents [26].

The second goal is to achieve an active WHR, which

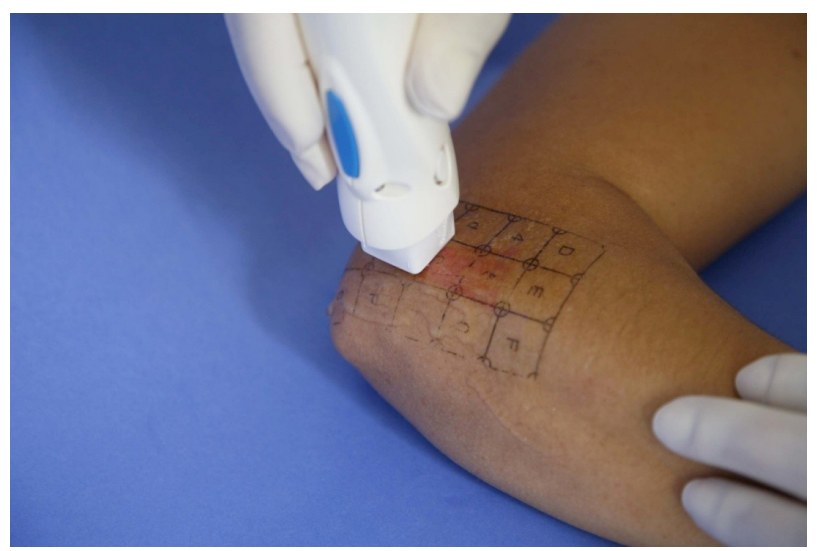

Figure 2. Direct contact between the active electrode and the skin is required. from a biological point of view should manifest by viable neural elements, normal vascularization, and collagen production. This is in contrast to yet prevalent therapeutic approaches, which includes the use of anti-inflammatory medications, local injections, and prolonged physical therapy [4].

\subsection{Alternatives for the Noninvasive Treatment of Tendinosis}

\subsubsection{Nonsteroidal Anti-Inflammatory Drugs}

Nonsteroidal anti-inflammatory drugs (NSAIDs) are commonly prescribed or acquired over the counter as the first attempt to curb symptoms while hoping that any inflammation present at the site will be resolved, and the symptoms will abate. However, it is understood that tendinosis is the result of a failed wound healing process mediated by the body's inflammatory response. Therefore, curbing the inflammatory response may in fact be detrimental. As a matter of fact, Almekinders et al. found that NSAIDs have potentially negative effects during the proliferative phase of healing since they are associated with decreased DNA synthesis [50].

\subsubsection{Steroid Injections}

The "Standard of Care" continues to be cortisone injections. These treatments are characterized by an early relief of pain in some patients but are followed by long term damage to the tendon and ligament structures. It has been clearly established that clinical outcomes of corticosteroid injections are worse than placebo at 26 and 52 weeks, as demonstrated by several randomized clinical trials and systematic reviews [51,52].

\subsubsection{Extracorporeal Shock Wave Therapy (ESWT)}

The capabilities of ESWT to break down calcium deposits seem to indicate that the ideal indication for this technology will reside in the treatment of calcifying tendinopathy. ESWT uses sound waves focused onto the deposit. In some German studies, 30\% - 70\% of patients obtained pain relief, and in $20 \%-77 \%$ of cases the calcium deposit disappeared or disintegrated [53]. In fact, ESWT was approved in the United States market only for the treatment of chronic proximal plantar fasciitis, in patients that have failed conservative therapies.

Current knowledge cannot support the use of ESWT for the treatment of noncalcified tendinosis. A recent systematic review concluded that there is platinum evidence that ESWT "provides little or no benefit in terms of pain and function in lateral elbow pain” [54-56]. Moreover, Staples et al. recently conducted a randomized clinical trial utilizing ultrasound guided ESWT and concluded that their findings did not support the use of ESWT for the treatment of lateral epicondylitis [57]. 


\subsection{Alternatives for the Invasive Treatment of Tendinosis}

\section{Surgery}

Surgical techniques deemed successful call for resection of the pathological tissue [58] including afferent nociceptors [27] and the induction of the WHR. RF based surgical procedures are the gold standard in the treatment of tendinosis as they have an antinociceptive effect [27] and trigger the WHR [25]. In fact in a randomized clinical trial Meknas et al. (2008) found that RF-based surgical procedures provided earlier pain relief and improved grip strength when compared with extensor tendon release and repair, another surgical modality that is deemed successful [43].

\section{Discussion}

The bases of the clinical application of monopolar, capacitive coupled radiofrequency technology are: its ability to raise the temperatures of tendons and ligaments to a point where collagen structures will denature [46], its ability to trigger the WHR [59], and still yet to be demonstrated, an antinociceptive effect and the stimulation of MPCs.

There are a number of pathological entities of the connective tissues characterized by a failed or nonsexisting WHR and in which the triggering of this physiological process is desirable. The histopathological characteristics of these entities are noninflammatory in essence with localized collagen damage, fibroblastic hyperplasia, and degenerative changes [8,9]. Typical examples of tendinosis and fasciosis can be found throughout the literature $[1-4,8,9,11,15,16]$. By delivering supra-physiological temperatures to connective structures, noninvasive mcRF seeks to induce thermal wounds, via electrocoagulation, while preserving the overlaying tissue (e.g., skin and subcutaneous layers) which results in a sought after wound healing response [5,7]. The clinical benefit of inducing the wound healing process is well established [6,10,12-14]. The cornerstone of the clinical significance of noninvasive mcRF resides in its ability to stimulate a WHR largely similar in nature and timing to those of incisional or burn wounds [7] in a totally noninvasive fashion, whereas other RF generators utilized for this purpose require an open or otherwise invasive surgical procedure.

Surgical procedures continue to be the last resort in the treatment of tendinosis [58] and should be reserved for the treatment of recalcitrant cases. Noninvasive mcRF offers a therapeutic alternative to the noninvasive standard of care-corticosteroid injections-since current knowledge does not support their use for these indications.

Noninvasive mcRF is safe; the rate of adverse events when used for non-orthopaedic applications is less than two per thousand cases, and are aesthetic and transitory in nature. To There have been no reported adverse events with the use of the technology for orthopaedic applications. Noninvasive mcRF will not interfere with other procedures. Should a surgical procedure subsequently become necessary after a treatment, it can be performed regardless of the time elapsed.

\section{Conclusion}

Noninvasive mcRF has the potential to become the treatment of choice for common tendinopathies and ligamentopathies. Additional clinical studies are necessary to understand the full potential of this novel technology.

\section{REFERENCES}

[1] L. Bernstein, “Tendinosis of the Shoulder," Mississippi Valley Medical Journal, Vol. 73, No. 6, 1951, pp. 155157.

[2] R. P. Nirschl and F. A. Pettrone, “Tennis Elbow. The Surgical Treatment of Lateral Epicondylitis,” Journal of Bone \& Joint Surgery, Vol. 61, No. 6A, 1979, pp. 832839.

[3] R. P. Nirschl, "Elbow Tendinosis/Tennis Elbow," Clinical Journal of Sport Medicine, Vol. 11, No. 4, 1992, pp. 851-870.

[4] B. S. Kraushaar and R. P. Nirschl, "Tendinosis of the Elbow (Tennis Elbow). Clinical Features and Findings of Histological, Immunohistochemical, and Electron Microscopy Studies," Journal of Bone \& Joint Surgery, Vol. 81, No. 2, 1999, pp. 259-278.

[5] N. Maffulli, K. M. Khan and G. Puddu, "Overuse Tendon Conditions: Time to Change a Confusing Terminology," Arthroscopy, Vol. 14, No. 8, 1998, pp. 840-843. doi:10.1016/S0749-8063(98)70021-0

[6] E. R. Cosman, B. S. Nashold and J. Ovelman-Levitt, "Theoretical Aspects of Radiofrequency Lesions in the Dorsal Root Entry Zone,” Neurosurgery, Vol. 15, No. 6, 1984, pp. 945-950. doi:10.1227/00006123-198412000-00035

[7] B. Avitall, M. Khan, D. Krum, et al., "Physics and Engineering of Transcatheter Cardiac Tissue Ablation,” Journal of the American College of Cardiology, Vol. 22, No. 3, 1993, pp. 921-932. doi:10.1016/0735-1097(93)90212-J

[8] B. Avitall, D. Lafontaine, G. Rozmus, et al., "The Safety and Efficacy of Multiple Consecutive Cryo Lesions in Canine Pulmonary Veins-Left Atrial Junction," Heart Rhythm, Vol. 1, No. 2, 2004, pp. 203-209. doi:10.1016/j.hrthm.2004.03.058

[9] B. Avitall, A. Urbonas, D. Urboniene, G. Rozmus and R. Helms, "Linear Lesions Provide Protection from Atrial Fibrillation Induction with Rapid Atrial Pacing,” Journal of Cardiovascular Electrophysiology, Vol. 13, No. 5, 2002, pp. 455-462. doi:10.1046/j.1540-8167.2002.00455.x

[10] E. Daoud and F. Morady, "Catheter Ablation of Ven- 
tricular Tachycardia,” Current Opinion in Cardiology, Vol. 10, No. 1, 1995, pp. 21-25. doi:10.1097/00001573-199501000-00005

[11] M. D. Lesh, "Interventional Electrophysiology-Stateof-the-Art 1993,” American Heart Journal, Vol. 126, No. 3, 1993, pp. 686-698. doi:10.1016/0002-8703(93)90419-A

[12] L. A. Saxon, J. M. Kalman, J. E. Olgin, M. M. Scheinman, R. J. Lee and M. D. Lesh, "Results of Radiofrequency Catheter Ablation for Atrial Flutter," American Journal of Cardiology, Vol. 77, No. 11, 1996, pp. 1014-1016.

[13] J. S. Brodkey, Y. Miyazaki, F. R. Ervin and V. H. Mark, "Reversible Heat Lesions with Radiofrequency Current. A Method of Stereotactic Localization," Journal of Neurosurgery, Vol. 21, No. 1, 1964, pp. 49-53. doi:10.3171/jns.1964.21.1.0049

[14] A. Moraci, C. Buonaiuto, A. Punzo, C. Parlato and R. Amalfi, "Trigeminal Neuralgia Treated by Percutaneous Thermocoagulation. Comparative Analysis of Percutaneous Thermocoagulation and Other Surgical Procedures," Neurochirurgia (Stuttg), Vol. 35, No. 2, 1992, pp. 48-53.

[15] W. H. Sweet and J. G. Wepsic, "Controlled Thermocoagulation of Trigeminal Ganglion and Rootlets for Differential Destruction of Pain Fibers. 1. Trigeminal Neuralgia,” Journal of Neurosurgery, Vol. 40, No. 2, 1974, pp. 143-156. doi:10.3171/jns.1974.40.2.0143

[16] M. H. Seegenschmiedt and R. Sauer, "The Current Role of Interstitial Thermo-Radiotherapy," Strahlentherapie und Onkologie, Vol. 168, No. 3, 1992, pp. 119-140.

[17] P. L. Privalov. Stability of proteins, "Proteins Which Do Not Present a Single Cooperative System," Advances in Protein Chemistry, Vol. 35, 1982, pp. 1-104. doi:10.1016/S0065-3233(08)60468-4

[18] C. A. Miles, T. V. Burjanadze and A. J. Bailey, "The Kinetics of the Thermal Denaturation of Collagen in Unrestrained Rat Tail Tendon Determined by Differential Scanning Calorimetry,” Journal of Molecular Biology, 27 Vol. 245, No. 4, 1995, pp. 437-446. doi:10.1006/jmbi.1994.0035

[19] A. Miniaci and M. J. Codsi, "Thermal Capsulorrhaphy for the Treatment of Shoulder Instability," The American Journal of Sports Medicine, Vol. 34, No. 8, 2006, pp. 1356-1363. doi:10.1177/0363546506287824

[20] A. M. Hill, I. T. Jones, U. Hansen, et al., "Treatment of Ligament Laxity by Electrothermal Shrinkage or Surgical Plication: A Morphologic and Mechanical Comparison,” Journal of Shoulder and Elbow Surgery, Vol. 16, No. 1, 2006, pp. 95-100.

[21] D. A. Coons and F. A. Barber, "Thermal Medial Retinaculum Shrinkage and Lateral Release for the Treatment of Recurrent Patellar Instability,” Arthroscopy, Vol. 22, No. 2, 2006, pp. 166-171. doi:10.1016/j.arthro.2005.08.043

[22] E. Farng, S. A. Hunt, D. J. Rose and O. H. Sherman, “Anterior Cruciate Ligament Radiofrequency Thermal Shrinkage: A Short-Term Follow-Up,” Arthroscopy, Vol. 21, No. 9, 2005, pp. 1027-1033. doi:10.1016/j.arthro.2005.05.015

[23] N. A. Darlis, R. W. Weiser and D. G. Sotereanos, "Partial
Scapholunate Ligament Injuries Treated with Arthroscopic Debridement and Thermal Shrinkage," Journal of Hand Surgery, Vol. 30, No. 5, 2005, pp. 908-914. doi:10.1016/j.jhsa.2005.05.013

[24] H. M. Kwon, B. K. Hong, G. J. Jang, et al., "Percutaneous Transmyocardial Revascularization Induces Angiogenesis: A Histologic and 3-Dimensional Micro Computed Tomography Study," Journal of Korean Medical Science, Vol. 14, No. 5, 1999, pp. 502-510.

[25] J. P. Tasto, J. Cummings, V. Medlock, R. Hardesty and D. Amiel, "Microtenotomy Using a Radiofrequency Probe to Treat Lateral Epicondylitis,” Arthroscopy: The Journal of Arthroscopic \& Related Surgery, Vol. 21, No. 7, 2005, pp. 851-860. doi:10.1016/j.arthro.2005.03.019

[26] K. M. Khan, J. L. Cook, N. Maffulli and P. Kannus, "Where Is the Pain Coming from in Tendinopathy? It May Be Biochemical, Not Only Structural, in Origin," British Journal of Sports Medicine, Vol. 34, No. 2, 2000, pp. 81-83. doi:10.1136/bjsm.34.2.81

[27] N. Takahashi, J. P. Tasto, M. Ritter, et al., "Pain Relief through an Antinociceptive Effect after Radiofrequency Application,” American Journal of Sports Medicine, Vol. 35, No. 5, 2007; pp. 805-810. doi:10.1177/0363546506297085

[28] C. F. Hyer, "Radiofrequency Microtenotomy of Plantar Fascia Is Effective, but Why?” Foot \& Ankle Specialist, Vol. 1, No. 6, 2008, pp. 368-369.

[29] L. J. R. Weil, J. P. Glover and L. S. S. R. Weil, “A New Minimally Invasive Technique for Treating Plantar Fasciosis Using Bipolar Radiofrequency: A Prospective Analysis,” Foot \& Ankle Specialist, 2008, Vol. 1, No. 1, 2008, pp. 13-18.

[30] N. T. Wright and J. D. Humphrey, "Denaturation of Collagen via Heating: An Irreversible Rate Process,” Annual Review of Biomedical Engineering, Vol. 4, 2002, 109-128. doi:10.1146/annurev.bioeng.4.101001.131546

[31] A. L. Fink, “Chaperone-Mediated Protein Folding," Physiological Reviews, Vol. 79, No. 2, 1999, pp. 425-449.

[32] M. Jaattela, "Heat Shock Proteins as Cellular Lifeguards," Annals of Medicine, Vol. 31, No. 4, 1999, pp. 261-271. doi:10.3109/07853899908995889

[33] R. S. Williams, J. A. Thomas, M. Fina, Z. German and I. J. Benjamin, "Human Heat Shock Protein 70 (HSP70) Protects Murine Cells from Injury during Metabolic Stress,” Journal of Clinical Investigation, Vol. 92, No. 1, 1993, pp. 503-508. doi:10.1172/JCI116594

[34] F. R. Sharp, S. M. Massa and R. A. Swanson, "HeatShock Protein Protection,” Trends in Neurosciences, Vol. 22, No. 3, 1999, pp. 97-99. doi:10.1016/S0166-2236(98)01392-7

[35] J. Bao, W. Naimark, M. Palasis, R. Laham, M. Simons and M. J. Post, "Intramyocardial Delivery of FGF2 in Combination with Radio Frequency Transmyocardial Revascularization," Catheterization and Cardiovascular Interventions, Vol. 53, No. 3, 2001, pp. 429-434. doi:10.1002/ccd.1196

[36] N. Yamamoto, A. Gu, C. M. DeRosa, et al., "Radio Frequency Transmyocardial Revascularization Enhances An- 
giogenesis and Causes Myocardial Denervation in Canine Model,” Lasers in Surgery and Medicine, Vol. 27, No. 1, 2000, pp. 18-28.

doi:10.1002/1096-9101(2000)27:1<18::AID-LSM3>3.0. $\underline{\mathrm{CO} ; 2-\mathrm{F}}$

[37] A. Hayashi, "Getting Athletes Back in the Game: A Global View,” AAOS Now, Vol. 2, No. 10, 2008, pp. 1-6.

[38] S. Yang, "Treatment Increases Survival after Transplantation Autologous Myoblasts Pigs in Skeletal Muscle,” University of Geneva, Geneva, 2005.

[39] D. Amiel, S. T. Ball and J. P. Tasto, "Chondrocyte Viability and Metabolic Activity after Treatment of Bovine Articular Cartilage with Bipolar Radiofrequency: An in Vitro Study," Arthroscopy, Vol. 20, No. 5, 2004, pp. 503510. doi:10.1016/j.arthro.2004.03.018

[40] J. P. Tasto, "The Role of Radiofrequency-Based Devices in Shaping the Future of Orthopedic Surgery," Orthopedics, Vol. 29, No. 10, 2006, pp. 874-875.

[41] J. P. Tasto and S. A. Ash, "Current Uses of Radiofrequency in Arthroscopic Knee Surgery," American Journal of Knee Surgery, Vol. 12, No. 3, 1999, pp. 186-191.

[42] E. Taverna, F. Battistella, V. Sansone, C. Perfetti and J. P. Tasto, "Radiofrequency-Based Plasma Microtenotomy Compared with Arthroscopic Subacromial Decompression Yields Equivalent Outcomes for Rotator Cuff Tendinosis,” Arthroscopy, Vol. 23, No. 10, 2007, pp. 10421051. doi:10.1016/j.arthro.2007.04.018

[43] K. Meknas, A. Odden-Miland, J. B. Mercer, M. Castillejo and O. Johansen, "Radiofrequency Microtenotomy: A Promising Method for Treatment of Recalcitrant Lateral Epicondylitis," The American Journal of Sports Medicine, Vol. 36, No. 10, 2008, pp. 1960-1965. doi:10.1177/0363546508318045

[44] B. D. Zelickson, D. Kist, E. Bernstein, et al., "Histological and Ultrastructural Evaluation of the Effects of a Radiofrequency-Based Nonablative Dermal Remodeling Device: A Pilot Study,” Archives of Dermatology, Vol. 140, No. 2, 2004, pp. 204-209. doi:10.1001/archderm.140.2.204

[45] R. A. Weiss, M. A. Weiss, G. Munavalli and K. L. Beasley, "Monopolar Radiofrequency Facial Tightening: A Retrospective Analysis of Efficacy and Safety in Over 600 Treatments," Journal of Drugs in Dermatology, Vol. 5, No. 8, 2006, pp. 707-712.

[46] T. Whipple and D. Villegas, "Non-Invasive Monopolar Radiofrequency Energy for the Treatment of Tendons and Ligaments: Temperatures Achieved and Histological Outcomes Richmond,” Orthopaedic Research of Virginia, 2009.

[47] C. Rose and N. Maffulli, "Non-Invasive Monopolar Capacitive-Coupled RF for the Treatment of Tennis Elbow: A Pilot Study,” 2009.
[48] F. Essers, "Non-Invasive Monopolar Capacitive Coupled RF: A Safety Study,” 2009.

[49] T. Weber and B. Kabelka, "Non-Invasive Monopolar RF for the Treatment of Tennis Elbow: 12 Months Follow-Up,” 2009 (In Preparation).

[50] L. C. Almekinders, A. J. Baynes and L. W. Bracey, “An in Vitro Investigation into the Effects of Repetitive Motion and Nonsteroidal Antiinflammatory Medication on Human Tendon Fibroblasts," The American Journal of Sports Medicine, Vol. 23, No. 1, 1995, pp. 119-123. doi:10.1177/036354659502300120

[51] L. Bisset, N. Smidt, D. A. Van der Windt, et al., "Conservative Treatments for tennis Elbow Do Subgroups of Patients Respond Differently?” Rheumatology, Vol. 46, No. 10, 2007, pp. 1601-1605.

[52] J. M. Bjordal, R. Lopes-Martins, J. Joensen, et al., “A Systematic Review with Procedural Assessments and Metaanalysis of Low Level Laser Therapy in Lateral Elbow Tendinopathy (Tennis Elbow),” BMC Musculoskeletal Disorders, Vol. 9, No. 10, 2008, pp. 9-75.

[53] M. Loew, W. Daecke, D. Kusnierczak, M. Rahmanzadeh and V. Ewerbeck, "Shock-Wave Therapy Is Effective for Chronic Calcifying Tendinitis of the Shoulder," The Bone \& Joint Journal, Vol. 81, No. 5, 1999, pp. 863-867. doi:10.1302/0301-620X.81B5.9374

[54] R. Buchbinder, S. Green, M. White, L. Barnsley, N. Smidt and W. J. Assendelft, "Shock Wave Therapy for Lateral Elbow Pain,” Cochrane Database of Systematic Reviews, Vol. 1, 2002, Article ID: CD003524.

[55] R. Buchbinder, S. E. Green, J. M. Youd, W. J. Assendelft, L. Barnsley and N. Smidt, "Shock Wave Therapy for Lateral Elbow Pain,” Cochrane Database of Systematic Reviews, Vol. 4, 2005, Article ID: CD003524.

[56] R. Buchbinder, S. E. Green, J. M. Youd, W. J. Assendelft, L. Barnsley and N. Smidt, "Systematic Review of the Efficacy and Safety of Shock Wave Therapy for Lateral Elbow Pain,” The Journal of Rheumatology, Vol. 33, No. 7, 2006, pp. 1351-1363.

[57] M. P. Staples, A. Forbes, R. Ptasznik, J. Gordon and R. Buchbinder, "A Randomized Controlled Trial of Extracorporeal Shock Wave Therapy for Lateral Epicondylitis (Tennis Elbow),” The Journal of Rheumatology, Vol. 35, No. 10, 2008, pp. 2038-2046.

[58] J. H. Dunn, J. J. Kim, L. Davis and R. P. Nirschl, “Ten- to 14-Year Follow-Up of the Nirschl Surgical Technique for Lateral Epicondylitis," The American Journal of Sports Medicine, Vol. 36, No. 2, 2008, pp. 261-266. doi:10.1177/0363546507308932

[59] L. J. England, B. M. Egbert and K. Pope, "Dermal Wound Healing in an Animal Model Following Monopolar Radio Frequency Treatment," Wound Healing Society Meeting, Chicago, 2005. 